\title{
Prevención del VIH: análisis de conductas sexuales de riesgo y propuestas de intervención.
}

\section{Cristina Giménez-García}

Salusex. Dpto. Personalidad, Evaluación y Tratamientos psicológicos. Universitat Jaume I. gimenezc@uji.es

Estefanía Ruiz-Palomino

Salusex. Dpto. Personalidad, Evaluación y Tratamientos psicológicos. Universitat Jaume I. eruiz@uji.es

Rafael Ballester-Arnal

Salusex. Dpto. Personalidad, Evaluación y Tratamientos psicológicos. Universitat Jaume I. rballest@uji.es

\section{RESUMEN}

La epidemia del VIH-Sida constituye uno de los problemas más importantes de salud pública. Sin embargo, los esfuerzos en el ámbito farmacológico, no se han acompañado de otros que mejoren la prevención comportamental. Por este motivo, se analizan variables cognitivas (percepción de gravedad y temor hacia el VIH, y de fiabilidad del preservativo), y conductuales (uso de método de barrera en relaciones estables, esporádicas y tras consumir sustancias) que incrementan las prácticas sexuales de riesgo para el VIH, así como algunas de las estrategias que han mostrado ser eficaces. Para ello, 805 jóvenes con edad promedio de 20,06 años $(\mathrm{DT}=2,49)(43,1 \%$ hombres y 56,9\% mujeres) cumplimentaron la Encuesta del Sida (Ballester et al., 2007) en 2019. A nivel general, en una escala de 0 a 100, se observa un promedio de temor percibido de 72,11 (DT=35,02) y una probabilidad percibida de 18,25 $(\mathrm{DT}=23,56)$, mostrando una fiabilidad percibida del preservativo de $2,65(\mathrm{DT}=0,52)$ en una escala de 0 a 3 . Respecto al uso sistemático de métodos de barrera, solamente un 32,2\% informa usarlo sistemáticamente en relaciones estables y un 56,6\% en parejas esporádicas, siendo un $38,1 \%$ el que lo hace tras consumir sustancias. Según el género, las mujeres muestran mayor promedio de temor percibido $(77,39$ vs 65,30 ; $t=-4,77, p=, 000)$ y probabilidad percibida $(20,06$ vs $15,88, \mathrm{t}=-2,49 ; \mathrm{p}=, 013)$, aunque dichas diferencias no resultan significativas en las conductas. Así pues, la población joven continúa presentando un perfil de riesgo con diferencias de género, en las que las mujeres siguen sin trasladar su probabilidad percibida y gravedad al plano conductual. Por ello, resulta necesario continuar con los esfuerzos preventivos que han mostrado eficacia, como aquellas propuestas multicomponentes que, desde una perspectiva de género, se han basado en teorías sociocognitivas y metodologías participativas.

Palabras clave: prevención conductual; VIH-Sida; método de barrera; factores de riesgo; actitudes. 


\begin{abstract}
The HIV-AIDS epidemic is one of the most important public health problems. However, pharmacological efforts have not been accompanied by efforts to improve behavioral prevention. For this reason, we analyze cognitive variables (perception of seriousness and fear of HIV, and reliability of condoms) and behavioral variables (use of barrier methods in stable and sporadic relationships and after substance use) that increase sexual practices at risk for HIV, as well as some of the strategies that have proven to be effective. For this purpose, 805 young people with an average age of 20.06 years $(\mathrm{SD}=2.49)$ (43.1\% male and $56.9 \%$ female) completed the AIDS Survey (Ballester et al., 2007) in 2019. At a general level, on a scale from 0 to 100, an average perceived fear of $72.11(\mathrm{SD}=35.02)$ and a perceived probability of 18.25 ( $\mathrm{SD}=23.56)$ were observed, showing a perceived condom reliability of $2.65(\mathrm{SD}=0.52)$ on a scale from 0 to 3 . Regarding the systematic use of barrier methods, only $32.2 \%$ report using it systematically in stable relationships and $56.6 \%$ in sporadic couples, with $38.1 \%$ doing so after consuming substances. According to gender, women show higher average perceived fear $(77.39$ vs. $65.30 ; \mathrm{t}=-4.77, \mathrm{p}=.000)$ and perceived probability ( 20.06 vs. $15.88, \mathrm{t}=-2.49 ; \mathrm{p}=.013)$, although these differences are not significant in the behaviors. Thus, the young population continues to present a risk profile with gender differences, in which women still do not transfer their perceived probability and severity to the behavioral level. Therefore, it is necessary to continue with preventive efforts that have shown effectiveness, such as those multicomponent proposals that, from a gender perspective, have been based on socio-cognitive theories and participatory methodologies.
\end{abstract}

Keywords: behavioral prevention; HIV-AIDS; barrier method; risk factors; attitudes. 


\section{INTRODUCCIÓN}

El abordaje de la salud y el bienestar de la población supone un reto complejo en el que la implicación de todos los agentes sociales resulta necesaria. En línea con acuerdos internacionales como la Declaración de Shanghai (Organización Mundial de la Salud, 2016) o los propios Objetivos de Desarrollo Sostenible - ODS (ONU, 2015), las instituciones de educación superior tienen, al menos, un triple reto en su desempeño. Por un lado, las Universidades tienen el cometido de mejorar las competencias técnicas y personales de futuros y futuras profesionales con el fin de que puedan promover los principios de la responsabilidad en su próximo desempeño laboral. Asimismo, y dada su función investigadora, las instituciones educativas superiores tienen la responsabilidad de generar y transferir un conocimiento riguroso que facilite caminos sostenibles para la consecución de los Objetivos de Desarrollo Sostenible. En tercer lugar, pero no menos importante, la Universidad debe suponer un agente promotor de salud que vele por el bienestar de la población.

De esta forma, y dada su naturaleza, la Universidad tiene tanto la responsabilidad como la capacidad de poner en marcha estrategias que promuevan la consecución de los ODS y mejoren el bienestar de la población. Para ello, resulta fundamental partir de un conocimiento empíricamente validado que permita analizar qué variables están modulando la calidad de vida de las personas, así como aplicar protocolos de evaluación e intervención pertinentes, eficaces y viables. Dentro de esta premisa, especialmente, cabría tener en cuenta la atención de aquellos colectivos más vulnerados y aquellas dimensiones de la salud que a lo largo de la historia han sido más estigmatizadas, como son la salud mental y la sexualidad. En este sentido, tal y como recoge el Observatorio de la Juventud en España (2020), la sexualidad supone una preocupación importante para los y las jóvenes a nivel nacional, que conviven con factores de riesgo como la desinformación, los sesgos actitudinales, las limitadas habilidades psicosexuales, la autoestima o las escasas habilidades para la gestión emocional, así como otras variables asociadas a los tabúes y estigmas que todavía acompañan a la sexualidad en nuestro marco cultural. Baste con un ejemplo: casi el $30 \%$ de los nuevos diagnósticos de infección por VIH en España han sucedido en jóvenes menores de 30 años (Plan Nacional sobre el Sida, 2020a) y el 27,1\% de casos de infección gonocócica, el 13\% de casos de sífilis, y el 38,7\% de casos de Chlamydia han sido diagnosticados en menores de 25 años (Plan Nacional sobre el Sida, 2020b). En esta línea, también se entenderían algunos resultados sobre infecciones de transmisión sexual de un estudio reciente, en contexto universitario, en el que solamente un 50\% de jóvenes decía emplear métodos de barrera, sistemáticamente, en relaciones sexuales esporádicas y un 35\% en las estables (UJI Hàbitat Saludable, 2018).

Teniendo en cuenta lo anterior y asumiendo los principios de la responsabilidad social universitaria, desde 1993 como equipo vinculado a la Universitat Jaume I y la Universitat de València y, desde 2014, como Centro Sanitario de Atención Psicológica reconocido por la Conselleria de Sanitat de la Generalitat Valenciana, el equipo de Salusex (www.salusex.uji.es) ha buscado, a través de la docencia, la asistencia, la investigación y la transferencia, la mejora de la calidad de vida sexual de la población. En dicha tarea, se ha concebido la importancia de abordar la salud sexual como un proceso multidimensional en el que resulta necesaria tanto la evaluación de los factores que favorecen las conductas de riesgo, como la valoración de la eficacia de aquellas intervenciones preventivas que se llevan 
a cabo. Desde este marco, en el presente trabajo se establecen dos objetivos. Por un lado, se busca analizar si todavía existe un perfil de riesgo para la exposición ante el VIH-Sida entre la población joven y, por otro, reflexionar sobre la experiencia del equipo Salusex relativa a la evaluación e intervención sobre los factores de riesgo asociados al VIH-Sida.

\section{EVALUACIÓN Y DIAGNÓSTICO DE VARIABLES DE RIESGO}

Tal y como se ha comentado, actualmente, el VIH-SIDA todavía representa una preocupación importante en la salud pública (UNAIDS, 2020). A pesar de algunos avances en los tratamientos médicos, las principales causas conductuales no han mejorado en la misma medida. En Europa, las conductas sexuales de riesgo son la vía de transmisión más elevada del VIH (Centro Europeo para la Prevención y el Control de Enfermedades, 2020). En España, donde la incidencia del VIH supera la media de las tasas europeas, el 83,1\% de los nuevos diagnósticos se basan en conductas sexuales de riesgo (Centro Europeo para la Prevención y el Control de Enfermedades, 2020; Centro Nacional de Epidemiología - ISCIII, 2020). Para abordar esta situación, resulta fundamental mejorar el conocimiento de los factores de riesgo que, en línea con la literatura (Ajzen, 1991; Amazaki \& Mori, 2011; Bandura, 1986; Cabral et al., 2004; Carvalho et al., 2015; Rosenstock et al., 1988), parecen modular la conducta sexual segura y, en concreto, el uso de métodos de barrera.

Los casi 30 años de experiencia investigadora y clínica en esta línea de trabajo han mostrado la diversidad de factores implicados en la prevención del VIH y la compleja interrelación que sucede en el contexto de la intimidad sexual (Ballester-Arnal, RuizPalomino, et al., 2017). Resulta relevante atender los déficits y dificultades que se pueden presentar en importantes factores cognitivos como la información veraz acerca de la infección por $\mathrm{VIH}$, la percepción de riesgo, las actitudes hacia los comportamientos sexuales seguros o la competencia percibida para enfrentarse a diferentes situaciones sexuales relacionadas con el uso de un método de barrera o el rechazo de una práctica sexual no segura. Sin embargo, no menos importante resulta entender que existen otros factores que pueden actuar como predisponentes, por ejemplo, la búsqueda de sensaciones sexuales, la autoestima o el valor otorgado a la salud (Ruiz-Palomino et al., 2020). Al mismo tiempo, otros estudios han mostrado la importancia de ciertas variables moduladoras para el uso del preservativo (Ruiz-Palomino et al., 2018) como, por ejemplo: el vínculo afectivo, el placer percibido en las prácticas sexuales de riesgo o el miedo a la evaluación negativa.

En este marco de trabajo, el equipo realiza un esfuerzo continuado por actualizar la información relativa a las conductas de riesgo y sus factores asociados, entendiendo la necesidad de contar con información reciente que permita valorar la necesidad de abordar las variables de riesgo en las estrategias de intervención o, en caso de que fuera necesario, priorizar aquellas que presentaran un mayor riesgo en la actualidad.

Para este fin, en 2019, se contó con la participación de 805 jóvenes con edad promedio de 20,06 años (DT=2,49), identificándose el 43,1\% como hombres y el 56,9\% mujeres y, según la orientación sexual, el 82,5\% como heterosexuales, el 13,2\% como bisexuales, el 2,9\% como homosexuales, el $1,1 \%$ como pansexuales y el $0,3 \%$ como asexuales. Todas las personas que participaron cumplimentaron la Encuesta del Sida (Ballester et al., 2007) en distintas actividades comunitarias, realizadas con motivo de la Conmemoración del Día Mundial del VIH. En concreto, la Encuesta del Sida (Ballester et al., 2007) evalúa las variables sociocognitivas asociadas con la exposición sexual al VIH (Ajzen, 1991; Bandura, 
1986; Rosenstock et al., 1988): información, creencias actitudinales y autoeficacia, intención de uso del condón, conducta sexual segura y discriminación hacia las personas que viven con el VIH. La Encuesta muestra una consistencia interna y una fiabilidad test-retest adecuadas .62 y .84 respectivamente.

A partir de este estudio y en relación con las creencias, los resultados mostraron un promedio de temor percibido de $72,11(\mathrm{DT}=35,02)$ y una probabilidad percibida de 18,25 $(\mathrm{DT}=23,56)$, en una escala de 0 a 100 . Asimismo, en una escala de 0 a 3 , se observó una media de percepción de fiabilidad del preservativo de 2,65 (DT=0,52).

Respecto al uso de métodos de barrera, solamente un 32,2\% informa usarlo sistemáticamente en relaciones estables y un 56,6\% en parejas esporádicas, siendo un $38,1 \%$ el que lo hace tras consumir sustancias. Según el género, las mujeres muestran mayor promedio de temor percibido $(M=77,39 ; \mathrm{DT}=31,93)$ frente a los hombres $(\mathrm{M}=65,30$; $\mathrm{DT}=37,60)$ observándose diferencias estadísticamente significativas $(\mathrm{t}=-4,77, \mathrm{p}=, 000)$. Asimismo, las mujeres informan de una mayor probabilidad percibida $(M=20,06$; DT=24,22) que los hombres $(\mathrm{M}=15,88$; DT=22,48), mostrando también diferencias estadísticamente significativas $(\mathrm{t}=-2,49 ; \mathrm{p}=, 013)$. Sin embargo, no existen tales diferencias en la percepción sobre la fiabilidad del preservativo entre hombres $(M=2,63$; $D T=0,55)$ y mujeres $(M=2,67$; $\mathrm{DT}=0,49)(\mathrm{t}=-1,07, \mathrm{p}=, 284)$. Las diferencias tampoco resultan significativas en cuanto al uso sistemático del método de barrera en las relaciones estables $(34,7 \%$ de hombres vs $30,1 \%$ de mujeres; $\left.\mathrm{Chi}^{2}=7,11 ; \mathrm{p}=, 068\right)$, relaciones esporádicas $(51,3 \%$ de hombres vs $61,1 \%$ de mujeres; $\left.\mathrm{Chi}^{2}=5,18 ; \mathrm{p}=, 159\right)$ y su uso tras consumir sustancias $(35,4 \%$ de hombres y $40,1 \%$ de mujeres; $\mathrm{Chi}^{2}=2,72 ; \mathrm{p}=, 436$ ).

En conjunto, se observa cómo todavía existe un perfil de riesgo entre la población joven ante la exposición al VIH y otras ITS. De hecho, casi el $40 \%$ de las chicas y el $50 \%$ de los chicos estarían expuestos a las ITS en las relaciones esporádicas, subiendo casi al $70 \%$ en ambos casos, dentro de las relaciones estables. Estos porcentajes no son mejores en cuanto al uso de alcohol y otras drogas; fenómeno que, en pasados estudios, ya ha mostrado la gran interferencia que genera para la realización de conductas sexuales seguras (Fairlie et al., 2018; Ramiro et al., 2015). Al mismo tiempo, en línea con lo que recoge la literatura, la exposición al riesgo parece ser mayor en aquellas relaciones estables en las que dada la percepción de confianza y seguridad, suelen presentar un patrón más resistente al uso de métodos de barreras frente al empleo de los métodos anticonceptivos (Pastor \& RojasMurcia, 2019; Pérez-Jiménez et al., 2009). Así pues, pese a que la fiabilidad percibida del método de barrera y el temor percibido puedan ser elevados, variables como la baja probabilidad percibida y otras, anteriormente comentadas, generan un perfil de riesgo que requiere la atención preventiva. Para mejorar esta situación, resulta necesario diseñar estrategias de intervención sostenibles que faciliten la realización sistemática de conductas sexuales saludables.

\section{PROPUESTA DE INTERVENCIÓN PREVENTIVA}

En línea con la Declaración Universal de los Derechos sexuales (WAS, 1997), desde el equipo Salusex se busca fortalecer las competencias de cuidado de salud de la población, incrementando el bienestar sexual y la prevención de posibles riesgos físicos y psicosociales. En este contexto, se han desarrollado distintas líneas de acción asumiendo la necesidad de trabajar, al menos, en dos niveles. De esta forma, se ha buscado una atención global a la 
población, con independencia de la etapa de cambio de conducta en la que se encuentre (Prochaska et al., 1992), pero teniendo en cuenta la particularidad de algunos colectivos como la población adolescente, el estudiantado universitario o la población adulta mayor, personas con diversidad funcional, el colectivo LGTBI o los trabajadores comerciales del sexo.

Por un lado, se han desarrollado acciones comunitarias con amplia cobertura y dirigidas a incrementar la concienciación y sensibilización sobre la importancia del cuidado de la salud sexual. En este contexto, las intervenciones se han dirigido a aquellas personas que, encontrándose en etapas de precontemplación o contemplación (Prochaska et al., 1992), no son conscientes del riesgo que podrían suponer las relaciones sexuales desprotegidas o, en caso de serlo, no tienen motivación para llevar a cabo conductas preventivas. Para ello, se han realizado distintas acciones comunitarias de manera presencial o a través de las redes sociales en las que, mediante metodologías participativas y basadas en el aprendizaje significativo, se ha buscado incrementar la mejora del conocimiento y de creencias ajustadas que favorezcan la decisión de usar métodos de barrera. A partir de este esfuerzo, más de 25.000 personas se han visto beneficiadas.

Por otro lado, se han llevado a cabo intervenciones centradas en la mejora y mantenimiento de competencias de cuidado. En este marco, las acciones se han dirigido a aquella población que, teniendo cierta motivación para el cambio, todavía no cuenta con herramientas suficientes para realizar la conducta preventiva y mantenerla. Todas estas intervenciones se han basado en una metodología participativa que, fundamentada en teorías sociocognitivas, ha demostrado facilitar mejores resultados (Ballester-Arnal, Gil-Llario et al., 2017). De esta forma, en las intervenciones se han incluido técnicas que, según nuestra propia experiencia (Ballester-Arnal et al., 2015), han mostrado ser más eficaces. En este sentido, la participación de una persona con VIH promovería más cambios en las actitudes, creencias e incluso conductas de las personas. Asimismo, tanto los juegos de roles sobre habilidades sociales y destrezas motoras, como los debates grupales (p. ej., el papel del placer en el uso del preservativo, el miedo a la evaluación negativa, etc.), también han demostrado incrementar variables tan fundamentales como la percepción de riesgo o la intención de conducta. Además, las charlas dialogadas, lejanas a las exposiciones unidireccionales en las que tradicionalmente se basaban las estrategias preventivas, también han mostrado una mayor eficacia. En estas intervenciones, en las que han participado más de 1.000 personas, se ha partido de un diagnóstico de la población participante, lo que ha posibilitado adaptar a sus necesidades, tanto las metodologías, como los contenidos y estrategias pedagógicas. Asimismo, tal y como hemos comprobado, el papel de las personas facilitadoras ha sido fundamental para la eficacia de cada intervención. En este sentido, más allá de características sociodemográficas como la edad o del nivel educativo, otras variables parecen resultar más exitosas en la configuración de un buen agente de salud. De hecho, en línea con nuestra experiencia (Giménez-García et al., 2018), tanto educadores pares como expertos podrían ser exitosos, siempre y cuando contaran con habilidades como la empatía, la capacidad de generar un marco de confianza o la flexibilidad para adaptar la intervención a las necesidades de la población participante.

\section{CONCLUSIÓN}


La experiencia que se relata en este artículo busca ilustrar la importancia de continuar con los esfuerzos preventivos en el ámbito de la infección por VIH y otras infecciones de transmisión sexual. Asimismo, a través de este análisis, se pretende enfatizar en la capacidad que tiene la Universidad y, por ende, los equipos de investigación que la conforman, de cumplir con el compromiso adquirido con los Objetivos de Desarrollo Sostenible (ONU, 2015).

En concreto, a partir de la experiencia desarrollada por el equipo Salusex durante las últimas tres décadas, se ha mostrado cómo la Universidad tiene la posibilidad de asumir la responsabilidad de ejercer su rol como promotora de salud y transformación social. Más allá de las cifras, los programas de intervención desarrollados y fundamentados por la evidencia científica han logrado mejorar las competencias de las personas con las que se ha trabajado (y se sigue trabajando) para que, de esta manera, recuperen su bienestar y sean autónomas en el cuidado de su salud afectivo-sexual. Más todavía, este trabajo también ha redundado en la mejora de su bienestar en general dado que las herramientas trabajadas son generalizadas a otros escenarios e indirectamente a otras personas.

Dicha labor ha sido fruto del esfuerzo continuado por generar un espacio de investigación y acción, que ha posibilitado detectar necesidades reales de conocimiento y mejorar nuestras propias estrategias de intervención. Así pues, por un lado, cabría ser consciente de que la población todavía presenta un perfil de riesgo ante la infección por VIH, materializado en actitudes desajustadas y, en particular, en el escaso uso sistemático de los métodos de barrera. De esta forma, parece evidente la necesidad de seguir fortaleciendo variables fundamentales como la autoeficacia (Ballester et al., 2013; Gil-Llario et al., 2019), la motivación o las habilidades sociales (Whiting et al., 2019), teniendo en cuenta aspectos tan fundamentales como el género o la orientación sexual (Giménez-García et al., 2020). Para ello, resulta fundamental aplicar estrategias de intervención empíricamente validadas que hayan mostrado su capacidad para promover conductas preventivas sostenibles en la población. Al mismo tiempo, será necesario que instituciones como la Universidad generen sinergias con otros agentes sociales, con el fin de crear entornos saludables en los que llevar a cabo dichas conductas sea posible.

\section{REFERENCIAS}

Ajzen, I. (1991). The theory of planned behavior. Organizational Behavior and Human Decision Processes, 50(2), 179-211. http://doi.org/10.1016/0749-5978(91)90020-T

Amazaki, M. \& Mori, K. (2011). Health Action Process Approach to condom use in university students. The Japanese Journal of Health Psychology, 24(2), 9-21. http://doi.org/10.11560/jahp.24.2_9

Ballester-Arnal, R., Ruiz-Palomino, E., \& Gil-Llario, M. D. (2017). Structural Equation Modeling Test of an Integrated Model of Spanish Youth's Condom Use. AIDS and behavior, 21(5), 1407-1416. https://doi.org/10.1007/s10461-016-1430-x

Ballester, R., Gil, M. D., Ruiz, E., \& Giménez, C. (2013). Autoeficacia en la prevención sexual del Sida: la influencia del género/Self-efficacy in sexual HIV prevention: the influence of gender. Anales de Psicología, 29(1), 76-82. https://dx.doi.org/10.6018/analesps.29.1.124601.

Ballester-Arnal, R., Gil-Llario, M., Giménez-García, C., \& Kalichman, S. (2015). What Works Well in HIV Prevention Among Spanish Young People? An Analysis of 
Differential Effectiveness Among Six Intervention Techniques. AIDS and Behavior, 19(7), 1157-1169. https://doi.org/10.1007/s10461-014-0863-3

Ballester-Arnal, R., Gil-Llario, M. D., Ruiz-Palomino, E., \& Giménez-García, C. (2017). Effectiveness of a Brief Multi-Component Intervention to HIV Prevention Among Spanish Youth. AIDS and behavior, 21(9), 2726-2735. https://doi.org/10.1007/s10461-017-1815-5

Bandura, A. (1986). Social foundations of thought and action: A social cognitive theory. Prentice-Hall.

Cabral, R. J., Cotton, D., Semaan, S., \& Gielen, A. C. (2004). Application of the transtheoretical model for HIV prevention in a facility-based and a community-level behavioral intervention research study. Health Promotion Practice, 5(2), 199-207. https://10.1177/1524839903258765.

Carvalho, T., Alvarez, M. J., Barz, M., \& Schwarzer. R. (2015). Preparatory behavior for condom use among heterosexual young men: a longitudinal mediation model. Health Education \& Behavior, 42(1), 92-99. https://10.1177/1090198114537066

Centro Europeo para la Prevención y el Control de Enfermedades (2020). HIV/AIDS surveillance in Europe 2020 (2019. [Archivo PDF]. https://www.ecdc.europa.eu/sites/default/files/documents/hiv-surveillance-report2020.pdf.

Centro Nacional de Epidemiología - ISCIII (2020). Vigilancia Epidemiológica del VIH y sida en España 2019: Sistema de Información sobre Nuevos Diagnósticos de VIH y Registro Nacional de Casos de Sida. [Archivo PDF]. https://www.isciii.es/QueHacemos/Servicios/VigilanciaSaludPublicaRENAVE/Enfe rmedadesTransmisibles/Documents/VIH/pdfs\%20y\%20protocolo/Informe\%20VIH SIDA_20201130.pdf

Fairlie, A. M., Garcia, T. A., Lee, C. M., \& Lewis, M. A. (2018). Alcohol use and alcohol/marijuana use during the most recent sexual experience differentially predict characteristics of the sexual experience among sexually active young adult drinkers. Addictive behaviors, 82, 105-108. https://doi.org/10.1016/j.addbeh.2018.02.027

Gil-Llario, M. D., Morell-Mengual, V., Ruiz-Palomino, E., \& Ballester-Arnal, R. (2019). Factorial Structure and Psychometric Properties of a Brief Scale of the Condom Use Self-Efficacy for Spanish-Speaking People. Health education \& behavior, 46(2), 295-303.https://doi.org/10.1177/1090198118806964

Giménez-García, C., Ballester-Arnal, R., Gil-Llario, M.D.; Ruiz-Palomino, E., \& MartínezGómez, N. (2020). Infecciones de transmisión sexual en población joven y su prevención: un análisis según la orientación del deseo sexual. International Journal of Developmental and Educational Psychology, 1(1), 155-165. https://doi.org/10.17060/ijodaep.2020.n1.v1.1771

Giménez-García, C., Ballester-Arnal, R., Gil-Llario, M. D., \& Salmerón-Sánchez, P. (2018). Peer-Led or Expert-Led Intervention in HIV Prevention Efficacy? A Randomized Control Trial Among Spanish Young People to Evaluate Their Role. Health Promotion Practice, 19(2), 277-286. https://doi.org/10.1177/152483991773396 
Observatorio de la Juventud de España (2020). La salud afectivo-sexual de la juventud en España. [Archivo PDF].

http://www.injuve.es/sites/default/files/adjuntos/2020/01/revista_injuve_123.pdf

Organización de Naciones Unidas (2015). Transformar nuestro mundo: la Agenda 2030 para el Desarrollo Sostenible [Archivo PDF]. https://www.un.org/ga/search/view_doc.asp?symbol=A/RES/70/1\&Lang=S

Organización Mundial de la Salud (2016). Declaración de Sanghai sobre la Promoción de Salud [Archivo PDF]. https://www.who.int/healthpromotion/conferences/9gchp/Shanghai-declarationfinal-draft-es.pdf.pdf?ua $=1$

Pastor, Y., \& Rojas-Murcia, C. (2019). Un estudio comparativo de la conducta sexual y la percepción de riesgo en dos cohortes de estudiantes universitarios españoles. Universitas Psychologica, 18(3), 1-14. https://doi.org/10.11144/Javeriana.upsy18$\underline{\text { 3.crsb }}$

Plan Nacional sobre el Sida (2020a). Vigilancia Epidemiológica del VIH y sida en España 2019 [Archivo PDF].

https://www.mscbs.gob.es/ciudadanos/enfLesiones/enfTransmisibles/sida/vigilancia /Informe_VIH_SIDA_20201130.pdf

Plan Nacional sobre el Sida (2020b). Vigilancia epidemiológica de las infecciones de transmisión sexual, 2018 [Archivo PDF]. https://www.mscbs.gob.es/ciudadanos/enfLesiones/enfTransmisibles/sida/vigilancia /Vigilancia_ITS_1995_2018_def.pdf

Pérez-Jiménez, D., Santiago-Rivas, M., \& Serrano-García, I. (2009). Comportamiento Sexual y Autoeficacia para la Negociación de Sexo Más Seguro en Personas Heterosexuales. Revista interamericana de psicología, 43(2), 414-424.

Prochaska, J. O., DiClemente, C. C., \& Norcross, J. C. (1992). In search of how people change. Applications to addictive behaviors. The American psychologist, 47(9), 1102-1114. https://doi.org/10.1037//0003-066x.47.9.1102

Ramiro, L., Windlin, B., Reis, M., Gabhainn, S. N., Jovic, S., Matos, M. G., Magnusson, J., \& Godeau, E. (2015). Gendered trends in early and very early sex and condom use in 20 European countries from 2002 to 2010. European journal of public health, 25(2), 65-68. https://doi.org/10.1093/eurpub/ckv030

Rosenstock, I. M., Strecher, V. J., \& Becker, M. H. (1988). Social learning theory and the Health Belief Model. Health education quarterly, 15(2), 175-183. https://doi.org/10.1177/109019818801500203

Ruiz-Palomino, E., Gil-Llario, M. D., Giménez-García, C., \& Ballester-Arnal, R. (2020). Explanatory Psychological Factors of Inconsistently Condom Use among Spanish University Students: Gender Differences. The Spanish journal of psychology, 23, e12. https://doi.org/10.1017/SJP.2020.14

Ruiz Palomino, E., Ballester Arnal, R, Giménez García, C., Gil Juliá, B., \& Gil Llario, M. D. (2018). Clinical profile associated with HIV risk in Spanish youth. International Journal of Developmental and Educational Psychology, 2(1), 387-395. https://doi.org/10.17060/ijodaep.2018.n1.v2.1247 
UJI Hàbitat Saludable (2018). Informe de estilos de vida entre el estudiantado de la UJI. Universitat Jaume I.

UNAIDS (2020). Prevailing against pandemics by putting people at the centre [Archivo PDF]. https://www.unaids.org/sites/default/files/media_asset/prevailing-againstpandemics_en.pdf

WAS (1997). Declaración de los Derechos Sexuales [Archivo PDF]. https://www.espill.org/wp-content/uploads/2016/01/Derechos-Sexuales-1997.pdf

Whiting, W., Pharr, J. R., Buttner, M. P., \& Lough, N. L. (2019). Behavioral Interventions to Increase Condom Use Among College Students in the United States: A Systematic Review. Health Education \& Behavior, 46(5), 877-888. https://doi.org/10.1177/1090198119853008 